\title{
A pervasive neural network based fall detection system on smart phone
}

\begin{abstract}
This paper presents a pervasive fall detection system on smart phones which can monitor the elderly activities and identifies the occurrence of falls. The proposed pervasive fall detection system was developed as a smart phone-based application under the name of Smart Fall Detection $\odot$ (SFD). SFD is a standalone Android-based application that detects the falls using proposed trained multilayer perceptron (MLP) neural network while utilizes smart phone resources such as accelerometer sensor and GPS. Data from the accelerometer are evaluated with the MLP to determine a fall. When neural network detects the fall, a help request will be sent to the specified emergency contact using SMS and subsequently whenever GPS data is available, the exact location of the fallen person will be sent. The SFD performance shows that it can detect the falls with the accuracy of $91.25 \%$.
\end{abstract}

Keywords: Pervasive fall detection system, smart phone-based application, accelerometer, trained MLP, GPS 